\title{
Los cibercafé como lugares de prácticas trasnacionales: El caso de la maternidad a distancia ${ }^{1}$
}

\author{
Carolina Stefoni ${ }^{2}$ \\ Universidad Alberto Hurtado, Santiago, Chile. Email: cstefoni@uahurtado.cl
}

\begin{abstract}
Resumen: Los cibercafé o locutorios corresponden a un tipo de negocios que se han ido expandiendo con fuerza en sectores donde se congrega un número importante de migrantes en diversas ciudades del mundo. Estos lugares son apropiados, utilizados y significados de distintas maneras por los usuarios. Este artículo es parte de una investigación mayor sobre la formación de un enclave de migrantes en el sector de Plaza de Armas, en el centro de Santiago (Chile). El foco de atención está puesto en los cibercafé como lugares donde se despliega el ejercicio de la maternidad a distancia, una de las prácticas transnacionales más estudiadas en el campo de las migraciones. Utilizando un enfoque etnográfico, el artículo analiza las prácticas cotidianas que realizan las mujeres para comunicarse con sus hijos y familias durante los fines de semana. Idas a llamar por teléfono y conversaciones utilizando un computador son prácticas que hacen parte de una rutina que organiza la vida de las mujeres inmigrantes con hijos en las ciudades de origen. Esta rutina va fijando un lugar desde donde se ejerce la maternidad trasnacional, y ello constituye uno de los contextos de resignificación que adquieren los cibercafés y que será analizado en el presente artículo.
\end{abstract}

Palabras clave: Lugares trasnacionales, migración en Chile, maternidades trasnacionales, cibercafé.

\section{The 'cyber cafes' as places of transnational practices: The case of distant motherhood}

\begin{abstract}
The cyber cafes or booths is a type of business that has been expanding strongly in sectors where a large number of migrants gather in cities around the world. These places are appropriated, occupied and signified in different ways by their users. This article is part of a larger investigation on the formation of a migrant enclave in the area of _Plaza de Armas in downtown Santiago (Chile). The focus is on the cafes as places where distance motherhood exercize is unfold, one of the most studied transnational practices in the field of migrations. Using an ethnographic approach, the article analyzes the daily practices that perform the women to communicate with their children and families during weekends. Going to perform telephone conversations and the use of computers are practices that are part of a routine that organizes the lives of immigrant women with children in the cities of origin. This routine is installing a place where transnational motherhood is exercised, and is one of the resignification contexts acquired by cafes, which will be discussed in this article.
\end{abstract}

Key words: Transnational places, migration in Chile, transnational motherhood, cyber cafe. 


\title{
Os cibercafé como lugares de práticas transnacionais: o caso da distância de maternidade
}

\begin{abstract}
Resumo: O cibercafé ou cabines são um tipo de negócio que têm vindo a expandir fortemente em sectores onde se reúne um grande número de migrantes em cidades ao redor do mundo. Esses lugares são apropriados e significados usados de maneiras diferentes pelos usuários. Este artigo é parte de uma investigação maior sobre a formação de um enclave migrante na área da Plaza de Armas, no centro de Santiago (Chile). O foco é sobre o cibercafé como lugares onde se desenrola o exercício da maternidade a distância, uma das práticas transnacionais mais estudadas no campo da migraciones. Utilizando uma abordagem etnográfica, o artigo analisa as práticas cotidianas que realizam a mulheres para se comunicar com seus filhos e famílias durante a semana. Idas às conversas telefônicas usando um computador são práticas que fazem parte de uma rotina que organiza a vida das mulheres imigrantes com crianças nas cidades de origem. Esta rotina é definir um lugar onde a maternidade transnacional é exercido, e este é um dos contextos da redefinição adquiridos por os cibercafés e será discutido neste artigo.

Palavras-chave: Lugares transnacionais, Migração no Chile, maternidade transnacional, cibercafé.
\end{abstract}

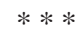

\section{Introducción}

Si bien la teoría diferencia y atribuye condiciones distintas a los procesos de globalización, de internacionalización, multinacionalidad y trasnacionalidad (Pries, 2005) en todos estos conceptos encontramos reflexiones que cuestionan el supuesto carácter desterritorializado de los mismos. Autores como Ay_e Ça_lar, Glick Schiller (2011) y Sassen (2001) entre otros, analizan el anverso de sistemas que se representan a través de metáforas como la aldea global o la Mc Donalización de las sociedades (Ritzer, 2000), en donde el flujo constante de información, capitales y estilos de vida, avanzaría permeando fronteras regionales y nacionales. La reflexión de estos autores reintroduce y visibiliza aquella dimensión que tiende a desaparecer detrás de las imágenes de lo ultra-globalizado, y ponen atención en los procesos de anclaje de lo global en territorios específicos, pues tal como señala M. Peter Smith (2003), lo global y lo trasnacional ocurren en lugares geográficos determinados. Sassen (2001) ya nos indicaba que la circularidad de capital e información no está distribuida homogéneamente en las ciudades o en los países, sino que existen zonas urbanas específicas donde se produce una alta circulación de información y capitales, lo que permite que lo global se imbrique con lo local. Siguiendo una línea similar, Glick Schiller y Ça_lar (2011) en el libro “Locating Migration”, analizan distintos casos en los que las prácticas trasnacionales de los grupos de inmigrantes van desplegándose en sectores urbanos específicos, manteniendo desde ahí, vínculos, redes y relaciones sociales con diversos otros lugares del planeta.

Este artículo forma parte de un estudio mayor realizado en Santiago centro, cuyo interés principal ha sido plantear que los lugares se pueden 
construir de manera trasnacional ${ }^{3}$. En términos muy sucintos se señala que la construcción del lugares se produce a partir de los significados que adquieren a) las prácticas sociales a través de las cuales el lugar es organizado, apropiado y habitado; b) la materialidad y artefactos que pueblan el lugar; y c) los vínculos sociales que mantienen los migrantes en el lugar, ya sea entre connacionales, con nacionales y con los compatriotas que viven en el país o comunidad de origen.

El carácter trasnacional de la migración puede desplegarse o no en cada una de las dimensiones señaladas, sin embargo, hay una práctica que destaca y que es el objeto de análisis de este artículo. Se trata de la maternidad a distancia ejercida por las mujeres que llegan cotidianamente a los cibercafés del caracol de calle Catedral, en el sector de la Plaza de Armas, en Santiago centro. La mayoría de los estudios sobre maternidad trasnacional se ha realizado desde la perspectivas de las mujeres o la familia (Schmalzbauer 2004, 2008; Bryceson y Vuorela, 2002; Hondagneu Sotello y Avila, 1997); desde una dimensión política (Herrera 2008, 2011; Sorensen 2008) y en algunos casos desde los hijos (Salazar Parreñas 2003; Takanishi, 2004). Sin embargo, este artículo busca comprender de qué modo se construye el lugar en el que cientos de mujeres ejercen la maternidad, aquel lugar semipúblico desde donde llaman y se comunican regularmente con sus hijos.

\section{Locutorios como espacios de conexión}

Los ciber café o locutorios corresponden a un tipo de negocios que se han ido expandiendo con fuerza en sectores donde se congrega un número importante de migrantes. Antes del repoblamiento del 'caracol de Catedral'4 , era posible encontrar en la misma calle a numerosos vendedores ambulantes de comida y tarjetas telefónicas. Sin embargo, las disposiciones de control desplegadas por la Municipalidad de Santiago a principios del 2000, obligó -o empujó- como señalan algunos entrevistados, a ingresar al 'caracol' y comenzar un proceso de formalización de sus actividades. En este contexto, son numerosos los centros de llamado y acceso a internet que se han ido instalando en el 'caracol', llegando a ser hoy en día más de 10 cibercafé en esta sola galería.

Sarah Lee (1999) realizó uno de los primeros estudios sobre los cibercafé como lugares de acceso público a internet. La autora a través del trabajo de campo en el sur-este de Inglaterra, analizó cómo era percibido el uso de internet en espacios públicos, en oposición a lo que se conocía sobre sus usos en espacios privados. Si bien el trabajo de campo lo realizó en un momento donde los cibercafé se estaban recién popularizando, resulta interesante observar que algunos de los elementos descritos en su investigación, como por ejemplo la diversidad de usuarios y variedad de usos que se les da a estos lugares, se mantienen e incluso profundizan en el caso de los cibercafé del 'caracol de Catedral'. En estos últimos es posible observar la presencia de inmigrantes hombres y mujeres provenientes de distintos paí- 
ses, padres y madres, jóvenes, turistas, chilenos, estudiantes, personas de la tercera edad, entre otros. Los clientes, por otra parte, utilizan y se apropian de internet y los equipos electrónicos de diversas maneras. En algunos casos son utilizados para mantener contacto con los hijos, con la pareja o con amigos, en otros, para conocer y desarrollar nuevas amistades a través de comunidades virtuales; también hay quienes van a buscar información específica o a desarrollar actividades de recreación tales como bajar y escuchar música. Estos usos también son mencionados en el estudio realizado por Martínez y Peñaranda (2011) en donde señalan que los cibercafé actúan como un espacio de encuentro entre inmigrantes, un lugar donde circula información de trabajos, arriendos y viajes, y donde se van desarrollando nuevas redes sociales (Peñaranda, Vitores et.al. 2011). De este modo, si bien los cibercafé del caracol están situados en un lugar en el que predomina la población inmigrante, la presencia de múltiples tipos de habitantes en el centro de Santiago determina que no sean sólo inmigrantes los clientes que acceden a estos locales, y que junto con esta diversidad de usuarios, se produzca una diversidad en las formas en que se utiliza internet. Dentro de la heterogeneidad de usos y clientes, he querido centrarme en las formas de uso que le dan las mujeres que llegan a estos lugares y la relación que ellas establecen con el local. Uno de los usos más frecuentes que ellas realizan es hablar con sus hijos y familiares durante los fines de semana. Así lo expresa un dueño de uno de los ciber del caracol:

"P: ¿Principalmente llaman a sus familias?

R: Sí, los días que más se llama son sábados y domingos

P: ¿Y para buscar pega (trabajo)?

R: No, más para hablar con la familia” (dueño ciber 2)

El trabajo de Lee también me parece relevante para el argumento que deseo explorar, ya que plantea la existencia de una relación entre determinadas prácticas y el lugar donde estas se ejecutan. Utilizando la distinción entre espacios públicos y privados, la autora argumenta que las formas que adquiere el uso de internet varia en dependencia de si se realiza en la casa o en un cibercafé. Ahora bien, sostengo que no es el lugar lo que determina la existencia de los distintos significados que puede adquirir las prácticas, sino el hecho de realizar en forma constante una misma práctica en un lugar determinado. Habitar el lugar implica por lo tanto sistematicidad, rutina y reiteración de las prácticas que ahí se realizan y sólo de este modo el lugar se vuelve propio y por tanto, único.

Siguiendo este planteo, el estudio buscó comprender cómo a través de las prácticas que realizan las mujeres en los ciber, se van estableciendo distintas relaciones con el lugar y con los dueños del local. Interesó también comprender los criterios de selección para escoger un cibercafé y cuáles son los significados que van adquiriendo estos lugares. Uno de los aspectos claves de estos significados, es el carácter trasnacional y de vinculación con lo que sucede en origen. 


\section{Materialidades presentes en los ciber}

La investigación utilizó una aproximación etnográfica y entrevistas a dueños de los locales y mujeres que van a realizar llamadas o utilizar internet. Las observaciones las realicé durante 2012 y se llevaron a cabo dentro de distintos cibercafés del caracol ubicado en calle Catedral, en el sector de Plaza de Armas (Santiago centro).

A través de las observaciones se distinguieron los siguientes artefactos y materialidades que van construyendo a los locales:

- Bienes materiales tecnológicos, tales como computadores, teclados, teléfonos, cámaras y micrófonos. Todos los ciber cuentan con computadores y cabinas telefónicas. Las diferencias se observan en el tiempo de uso y calidad de los mismos, por ejemplo, en algunas ocasiones los teclados están tan gastados que las letras prácticamente han desaparecido y las teclas se quedan pegadas al utilizarlas, lo que dificulta la escritura. Respecto de las cámaras y micrófonos, no todos los locales cuentan con estos implementos, pues en algunos casos prefieren no disponer de ellos porque desincentivaría el uso de teléfonos, que es finalmente desde donde se obtienen mayores ganancias los dueños de estos locales.

- Mobiliario con el que cuentan los ciber. La mayoría de los locutorios son pequeños, por lo que no caben más de 10 terminales. Muchos de ellos cuentan con alrededor de 5 terminales más 3 ó 4 cabinas telefónicas. En estos espacios por lo tanto, no es posible incorporar demasiados muebles, por lo que se reduce a sillas, mesones donde se ubican los computadores y cabinas para las llamadas. Las diferencias entre un local y otro está en el espacio disponible entre computador y computador, el aislamiento que presentaban las pantallas entre un usuario y otro (por ejemplo, si hay separadores entre pantallas), el tipo de sillas (de palo, con o sin respaldo, con cojín, etc.), y el tipo de cabinas (con puertas transparentes, aislación acústica, si la puerta puede o no cerrarse bien).

- Decoración. La decoración del lugar, los colores, la elección de los elementos que se promocionan, que se cuelgan en las paredes, que se pegan en las vitrinas permiten no sólo diferenciar a uno de otro local, sino que son elementos que apelan a procesos de identificación del lugar con una nacionalidad. En uno de los locales donde se realizó la observación, la pared tenía pintada tres banderas, una de Perú, otra de Ecuador y una tercera de Chile. En otro local ubicado fuera del 'caracol' (Punto Perú) hay mayor referencia a ese país, ya que las paredes están pintadas de color rojo y el techo blanco y hay promociones de tarjetas telefónicas que aluden a esa nacionalidad (Perú global, Hable más, Tarjeta internacional, El señor de los milagros, Hola mi Perú). En este sentido, el uso de banderas, propaganda alusiva a productos de un país determinado y en ocasiones la 
música o los protectores de pantalla seleccionados, van contribuyendo a una identificación del lugar con un país determinado.

- Productos adicionales que son vendidos en los locales. Los cibercafé incorporan la venta de una serie de elementos adicionales vinculados con los servicios que ofrecen -por ejemplo tarjetas telefónicas, chips para celulares, audífonos, micrófonos, envoltorios de celular, mouse, pilas, enchufes y cables- y con posibles demandas que pueden tener los clientes cuando utilizan los computadores (papas fritas, dulces, bebidas). Si bien en todos los locales hay algo adicional que se vende, no en todos ellos hay la misma oferta. En algunos sólo venden golosinas y en otros hay mesones y vitrinas con un mayor número de productos disponibles.

- Condiciones de acceso. Finalmente los cibercafé cuentan con un sistema tecnológico que permite ofrecer el servicio: velocidad de banda ancha, telefonía por internet, telefonía a través de convenios con compañías, telefonía por celular, etc. Se observa dos tipos de sistemas más frecuentes: contratos con compañías de telefonía para conexión a internet y sistema IP para llamadas telefónicas, lo que en algunos casos en contratado desde otro país ya que los costos son más económicos.

Si bien todos estos elementos están presentes en los locutorios, no todos son significados de la misma manera por los usuarios. En la elección del lugar por ejemplo, hay distintos criterios en función de lo que ofrecen los locales. Para algunos la velocidad de la conexión es clave, para otros es la tranquilidad para poder hablar, para otros conocer y tener confianza con el dueño y para otros la tarifa más económica.

"R: Pero especialmente vengo a este...

$\mathrm{P}: \dot{¿} Y$ porque prefiere venir a este local?

$\mathrm{R}$ : Porque este local está siempre uno más tranquilo, como que hay espacio, sobre todo por eso, porque hay espacio" (mujer usuaria de ciber)

"P. ¿ ¿Vienes siempre a este local?

R. Si paro aquí, por lo que el dueño es mi amigo, pero a cuando necesito otras cosas voy a otro local, pero más paro acá”. (mujer joven usuaria ciber)

Al comenzar a analizar las relaciones sociales que se van desplegando dentro de los locutorios, es posible identificar al menos cuatro tipos de relaciones y vínculos: en primer lugar, la relación entre la persona (cliente o usuario) y aquella con quien se comunica en el extranjero, sea telefónicamente o por computador, sea una persona o una red de personas; en segundo lugar la relación de la persona con la materialidad del lugar (por ejemplo como utiliza y se apropia de la tecnología disponible); en tercer lugar, las relaciones sociales que establece presencialmente con el 
administrador o dueño del local o bien con otros clientes que están en ese momento ahí, y finalmente la relación entre la persona y el lugar en su conjunto. Estas relaciones varían según el género, la edad y la condición migratoria que enfrenta la persona, por ejemplo, si el usuario tiene a su familia en Chile, el uso que realice del cibercafé, las relaciones con otros clientes, con el administrador, con quienes converse y con el lugar, serán distintas a una persona que tiene a sus hijos en Perú y debe enviar constantemente remesas para su mantenimiento. A su vez, los usos y relaciones que establece una mujer con sus hijos en Perú, es distinto a las formas de uso y relaciones que establece un hombre que también mantiene a su familia e hijos en el país de origen. Analizaré a continuación el caso de las mujeres que utilizan el ciber para comunicarse con su familia directa que está en origen.

\section{El lugar en la reproducción de las maternidades transnacionales}

En el contexto actual de creciente feminización de la migración laboral, no es de extrañar la presencia de mujeres en los locutorios que llaman y conversan con sus hijos y familiares. Comunicarse con ellos periódicamente permite mantener los vínculos, continuar ejerciendo el rol de madres y sostener, a partir de ello, la conformación de la familia (Bryceson y Vuorela, 2002; Schmalzbauer, 2004, 2008, Parella, 2007).

La presencia de mujeres que trabajan en otros países y que con ello logran sustentar a su familia, se repite en diversas partes del mundo. Ello ha abierto el análisis sobre el desarrollo de nuevos tipos de familias caracterizadas por la rearticulación de las relaciones sociales a través de las fronteras nacionales, es decir, la generación de estrategias y mecanismos que permiten mantener los vínculos sociales de modo de continuar siendo una familia pese a la distancia. Bryceson y Vuorela (2002) definen a la familia trasnacional como aquella cuyos miembros viven alguna parte (o gran parte) del tiempo separados y que son capaces de crear vínculos y generar mecanismos de negociación que permiten que sus miembros continúen sintiéndose parte de una unidad y perciban su bienestar desde una dimensión colectiva, pese a la distancia. Desde la perspectiva de los estudios de género, la familia trasnacional surge a partir de la reconfiguración del sistema de producción y reproducción en contextos de globalización (Parella, 2007). Si el proceso de industrialización se caracterizó por una separación entre la producción (ámbito masculino determinado por el trabajo remunerado realizado fuera de la casa) y la reproducción (ámbito femenino del cuidado y entrega de afecto realizado dentro del hogar), el proceso de globalización económica genera la necesidad de articular estos sistemas en un nuevo escenario. Tal como sostiene Parella (2007), frente a la globalización económica y laboral, es esperable que la reproducción y el cuidado también experimenten procesos de globalización. 
En este contexto, hemos observado en las últimas décadas cómo algunos miembros de familias se ven obligados/as a emigrar como estrategia para diversificar las posibilidades de sobrevivencia. Quién emigra, dónde y cómo se decide, dependerá de las formas en cómo la clase, el género y la etnia, estructuran a la familia y a la comunidad (Pessar y Mahler, 2003). En este sentido es central reconocer que las familias trasnacionales también se constituyen en distribuciones desiguales de poder, recursos y movilidad.

La emigración de mujeres y la consecuente salida de su entorno familiar, introduce tensiones a los modelos de maternidad tradicional (occidental y de clase media) establecidos culturalmente y que prescriben las formas de ser madre de las mujeres inmigrantes (Hondagneu-Sotello y Avila, 1007). En América Latina la iglesia católica ha jugado un rol central en la definición de lo que es la maternidad y cómo debe ser vivida. Bajo esta tradición se define que el lugar de las madres es al lado de su esposos e hijos, mientras que el padre es quien debe proveer de los recursos necesarios para la reproducción de la familia. Las mujeres inmigrantes que salen a trabajar para poder mantener a su familia subvierten este orden, lo que las transforma en objeto de cuestionamiento y crítica incluso a nivel político. Al respecto Salazar Parreñas (2003) analizó el caso de estigmatización y culpabilización a las que fueron sometidas las mujeres filipinas que tuvieron que emigrar para poder sostener a sus familias en la década de los noventa. Los medios de comunicación e incluso el Presidente de la República de la época, Fidel Ramos, insistieron sistemáticamente en los costos que debía asumir la familia y los hijos cuando la madre se ausentaba. Este discurso, sin embargo, se suavizó cuando se tomó conciencia del enorme aporte económico que hacían estas mujeres a la economía nacional a través del envío regular de remesas.

Cuando las mujeres alteran este orden de género, quedan expuestas a descalificaciones y cuestionamientos a su condición de madres, llegando en muchos casos a ser acusadas de madres abandonadoras y despreocupadas de sus hijos. Este estigma puede reproducirse en los niños que quedan en origen, siendo también calificados de hijos abandonados por la comunidad (Takanishi, 2004). El cuestionamiento social implica que en muchas mujeres la distancia y lejanía no se traduce sólo en nostalgia, añoranza y dolor por la separación, sino en una culpa que las acompaña y que durante su experiencia migratoria buscarán conciliar de algún modo.

La maternidad trasnacional surge entonces a partir de la reconfiguración de lo productivo y reproductivo en un contexto transfronterizo. La mujer debe salir a buscar trabajo para asegurar el sustento familiar sin que ello signifique renunciar a la responsabilidad que tiene como mandato cultural en el cuidado, protección y entrega de afecto a los miembros de su hogar (Schmalzbauer, 2004, 2008). La distancia física que supone la migración obliga a buscar mecanismos que permitan continuar con el ejercicio de ambos roles, lo que explica la significativa presencia de mujeres en los locutorios, pues las llamadas telefónicas les permiten estar allá a la vez que trabajan en un lugar distante para poder asegu- 
rar el mantenimiento del hogar. Hondagneu-Sotello y Avila (1997) señalan que las mujeres inmigrantes deben 'estar aquí, pero 'estar allá' a la vez, pues es la única forma de seguir brindando protección, cariño y cuidado estando a miles de kilómetros de distancia. En el estudio de Catedral, el dueño de un ciber señala una frase que refuerza la idea de mujeres situadas simultáneamente en dos lugares para continuar ejerciendo el rol de madres.
"R: Son diferentes caso, pero el mismo fondo, todos tienen el mis- mo fondo.
$\mathrm{P}:$ y cuál es ese fondo?
R: El problema familiar, que el hijo que no estudió, que le faltó. Es que es como si estuvieras en la casa, pero llamándolo, que por qué no estudiaste, que por qué no hiciste eso. O cogieron el dinero, o no lo utilizaron para lo que debiera de ser. Es todo el mismo fondo. Y también es oír desgracias no, que no faltó el sinvergüenza del hijo que se llevó la plata que la mamá le mandaba y al final chocó con la plata y a la final no estuvo no ahí con ellos
P: pero es la familia entonces
R: es la familia, todo es la familia”. (dueño ciber 1)

La emigración masculina, en cambio, es vista como una extensión en su rol de proveedores de recursos económicos, por lo que en ellos no se instala la tensión que significa tener que 'seguir allá', pues el ámbito de lo doméstico y del cuidado no ha sido definido culturalmente como su espacio ni responsabilidad. Esto por cierto que no significa que no extrañen a sus familias, pues muchas veces refieren al dolor que significa estar lejos de los seres queridos, pero al no ser los responsables directos de su cuidado, la emigración no es cuestionada socialmente ni pone la presión emocional que significa para las mujeres estar lejos de sus hijos. Estas distintas formas de vivir la migración según el género se traducen en prácticas diferenciadas al momento de ir al locutorio a llamar. El dueño de un ciber señala una de estas diferencias al plantear que los hombres hablan más corto, que entregan la información ‘y listo', y que no suelen conversar demasiado largo con la familia.

“P. y en el caso de los hombres, también es la familia con la que hablan?

R. si, con la familia, pero vienen y mamá, ya te mandé la plata y chao (risas)” (entrevista dueño ciber 1 )

La construcción de la maternidad y familia trasnacional requiere de una comunicación constante, pues es vital para la reproducción del vínculo entre los distintos miembros. Senyurekli (2008) señala que los medios a través de los cuales se mantienen los vínculos más allá de las fronteras son los medios tecnológicos de comunicación (teléfono, internet, celular, entre otros); el envío de remesas económicas y remesas sociales, tales como ideas, comportamiento, identidades y capital social, los que van transformando la vida social y política de las personas en origen y destino (Levitt, 1998) y las posibles visitas que realicen a su lugar de origen, lo que dependerá entre 
otras cosas, del estatus migratorio, de la disponibilidad de recursos, del género y de las posibilidades laborales.

De este modo, las llamadas telefónicas, llamadas por internet, envío de remesas sociales y económicas, así como posibles viajes a los lugares de origen, son prácticas que permiten a las mujeres inmigrantes en Chile mantenerse conectadas y vinculadas con sus hijos que están en origen, y con ello, continuar ejerciendo el rol de madres mientras están lejos. Esperar sin embargo, que las relaciones se mantengan igual que cuando se comparte el mismo espacio, es ilusorio. Las relaciones intrafamiliares experimentan importantes transformaciones cuando la familia se separa. La distancia va redefiniendo los roles de cada uno de los miembros e incluso puede incorporar nuevos integrantes como es el caso de los abuelos que cuidarán a los niños. Estas transformaciones estarán a su vez en dependencia de los contextos sociales, culturales y económicos que tengan las familias a ambos lados de la frontera (Parrado y Flippen, 2005).

En el contexto de la construcción transnacional de la familia y el ejercicio trasnacional de la maternidad, quisiera llamar la atención sobre el hecho de ir al locutorio a llamar, de hacerlo de manera frecuente, sistemáticamente una o dos o más veces por semana. Planteo que la importancia de ir a llamar es tan central, que se constituye en una práctica que organiza el tiempo y el espacio de la experiencia migratoria, particularmente en el caso de la mujer.

"Cada domingo que yo salgo llamó a la casa y a mi pareja también. Esa es la única forma y decir que estoy bien y como están allá” (entrevista mujer peruana)

Esperar el domingo, dejar separado algo de dinero para la llamada, pensar lo que se dice, pensar en las preguntas, caminar hasta el lugar donde se realiza la llamada, marcar, escuchar las voces, conversar con una, dos o más personas, despedirse, pagar. Este conjunto de prácticas se van convirtiendo en un ritual que organiza parte importante de la vida de las mujeres inmigrantes. Es un ritual que permite poner una certeza dentro de un continuo nostálgico, es la única forma, dice la mujer en la entrevista.

En este ritual se suele volver al mismo lugar para realizar las llamadas, ello puesto que la identificación de un lugar es parte de la ritualización de la práctica. Las razones para escoger uno u otro lugar son variadas: cercanía, comodidad, mayor espacio, tranquilidad, buenas tarifas o bien, la buena relación con el administrador o dueño. No hay un lugar correcto, sino simplemente un lugar desde donde se establecen los vínculos con los otros miembros de la familia.

Las mujeres utilizan múltiples formas para comunicarse, por ejemplo, cargar el celular con una tarjeta, lo que les da libertad para llamar desde cualquier lugar, pero cuando no queda crédito o se quiere hacer llamadas más económicas, van a un locutorio. Aquí también pueden usar 
internet para enviar un correo o comunicarse por chat o skype (o similar). La ventaja de internet es que les permite ver a la familia y a los hijos. Sin embargo, ello requiere de cierta experticia en el uso del teclado y de que la familia en origen disponga de acceso a internet en la casa.

Ir al locutorio de manera habitual entrega información respecto de cómo las mujeres usan el espacio público (calle Catedral) y los lugares semipúblicos como el ciber café. La calle Catedral genera en varias mujeres sospecha, no les agrada ver a las personas ahí sentadas, 'esperando que les llegue un trabajo, ${ }^{5}$. Es por ello que se aproximan a este lugar en forma más utilitaria, se trata de ir a llamar y regresar, entrar y salir, no quedarse, pues hay temor a ser confundida con aquello que rechazan. A diferencia de los hombres que habitan la esquina y que la han hecho suya ${ }^{6}$, la relación de algunas mujeres con este lugar es de tránsito, de pasada, tiempos cortos y breves cargados se emoción y significados, pero que no le permiten adueñarse de lo público.

"P: Y bueno, en relación a la calle Catedral, viene frecuentemente, ¿le gusta venir para acá?

R: No

P: No le gusta

R: No, no me gusta para nada, yo vengo por los llamados, por internet y por nada más” (entrevista Jennifer, ciber).

"R: Aquí en Nataniel, no salgo, yo paso encerrada de mi trabajo a mi casa, de mi casa al trabajo o si no me voy donde mi amiga que vive en Cerrillos” (entrevista Juana, ciber)

Dada la importancia que reviste para las personas ir a un cibercafé a llamar a sus seres queridos, resulta central comprender que los medios que comunican (teléfono, remesas, viajes) van adquiriendo significados distintos en la medida en que favorecen o tensionan las relaciones. Por ejemplo, el estudio de Madianou y D. Miller (2011) sobre el uso de telefonía celular que utilizan mujeres filipinas emigrantes para comunicarse con sus hijos, especialmente a partir de la masificación del producto y su abaratamiento en los costos, plantea que el significado otorgado al celular varía considerablemente entre madres e hijos. Si para las primeras es un elemento que permite seguir en contacto cotidiano con los hijos, no perder de vista lo que hacen, cómo están ni lo que quieren, para los hijos es percibido como algo invasivo, pues los llaman cuando no necesariamente quieren hablar, y sienten que no favorece una comunicación más personal, sino sólo utilitaria e informativa. El significado de las remesas también es ambivalente, pues si bien son parte central de la justificación del proyecto migratorio, también se transforman en un mediador de las relaciones entre los miembros de la familia.

"Igual mantengo una relación, la llamo, sino la llamo yo, me llama ella porque necesita plata. Lo que es así lamentablemente, pero tiene 12 años, ya pide sus cosas, ya está como una señorita, papá me falta esto" (entrevista Luis B. locatario caracol). 
De este modo, las reflexiones en torno a la materialidad realizadas por Daniel Miller y Madianou (2011), permiten una aproximación distinta a las TICS, no ya como medios ultrasofisticados que permiten la comunicación instantánea anulando la distancia espacial, sino comprender de qué modo éstos son apropiados, resignificados y utilizados por parte de los y las migrantes.

Retomando el trabajo de Lee que señalaba al comienzo de este apartado, hay una diferencia significativa que surge a partir del análisis de los centros de llamado y locutorios en el centro de Santiago. Para la autora los cibercafé juegan un rol central en el mantenimiento de los vínculos con el lugar de origen. Lee señala que en aquellos casos donde el hogar estaba lejos (ya sea porque se trataba de turistas o personas que habían llegado a trabajar a la ciudad dejado parte de su familia en origen), el uso del correo electrónico les permitía renovar el vínculo con la familia y actualizar el sentido local de pertenencia. Ello quiere decir que a través de la comunicación por internet, la persona podía seguir formando parte del lugar donde pertenecía, sin necesidad de estar físicamente ahí. ¿Es esto similar a la experiencia de estar ahí que buscan las madres y los inmigrantes al llamar por teléfono o comunicarse por internet? Considero que hay una diferencia muy importante, pues no se trata de actualizar un sentido de pertenencia, sino de producir un nuevo sentido de pertenencia multilocal. Conectarse o llamar por teléfono recuerda que no se está ahí, pero a través de la habitualidad y rutinización del llamado, se logra estar de una manera distinta, una manera que está dada por la simultaneidad del estar aquí y allá. Esta lectura es posible en la medida en que se incorpora el enfoque trasnacional, pues permite ir más allá de la idea de que la comunicación por internet reproduce el vínculo social a pesar de la distancia, lo que mantendría un sentido de pertenencia con la comunidad de origen. El enfoque trasnacional permite comprender que el locutorio es un lugar donde se despliegan distintas escalas geográficas (local, nacional, regional) y sociales (personal, familiar, comunitaria) en donde cada migrante, desde su computador o teléfono, las transita y articula de múltiples maneras.

\section{Diversos usos, prácticas y significados del locutorio}

Hay otras formas de uso asociados a distintos tipos de experiencias migratorias, como por ejemplo aquellas donde se ha producido la reunificación familiar o han formado familia en Chile. En estos casos, la comunicación con el país de origen suele ser más esporádica, y está asociada en muchos casos a fechas importantes como navidad, día de la madre o cumpleaños de parientes o amigos específicos. También se comunican por temas puntuales, como la enfermedad de algún familiar o la toma de decisiones que competen a toda la familia. La menor frecuencia de llamadas dificulta o imposibilita el desarrollo del ritual presente en el caso de las madres migrantes con hijos en origen, por lo que elección del lugar es más azarosa o los criterios pueden cambar con mayor facilidad. 
Otra forma de uso de los cibercafé es la que realizan personas más jóvenes. En estos casos los usos están asociados al entretenimiento, a 'despejar la mente’. Para ello suelen bajar y escuchar música, chatear con amigos y conocer nuevos amigos a través de las redes sociales.

"P. El computador cuando lo usas, en qué lo usas.

R. Lo uso verificando mensajes, si me han enviado algún mensaje de mi trabajo o de mi país. De ahí buscar alguna información de auto motivación o escuchar música, mas para despejar mas que todo, más que eso no.

(...)

P. ¿Y usas el Chat?

$\mathrm{R}$. Si, lo use en un determinado tiempo, como estoy en un país ajeno, entonces para puro despejarme, pero como puro entretenimiento pero no para pegarme o estar metida” (entrevista mujer ciber).

En algunos casos, especialmente las personas más establecidas o con mayores recursos, arriendan servicio de internet en la casa. En estos casos la comunicación con la familia que está en origen se produce desde el espacio privado. Aun así, es posible identificar diversas formas de utilizar la tecnología para mantenerse conectados con los familiares. Un caso que visualiza el dinamismo entre el uso de las tecnologías y la reproducción de los vínculos familiares, es la experiencia relatada por Carlos.

"R. Yo entro a Internet todos los días a revisar los correos. Durante la semana estoy conectado 3 o 4 veces en la semana. Lógico que cuando hacen algo indebido, no les gusta que los esté retando, pero yo les digo. Hablo con mi hija y con el pequeño. Dejamos el internet prendido, yo veo si pasa, cuando está si está jugando. Ellos también me ven cuando yo estoy en la casa, descansando" (Carlos).

El entrevistado cuenta que prende el skype y pone su computadora en el living. En su casa en Perú, sus hijas hacen lo mismo y cada quien sigue con su rutina durante horas. Como ambos computadores están conectados a la vez, cada cierto tiempo se preguntan cosas, comentan noticias y cosas que les pasan en el día. Es como tenerlos invitados en la casa y conversar libremente, sin el 'formato' que implica sentarse en un lugar frente a la pantalla y conversar. La disponibilidad permanente de acceso, como es el caso de Carlos, le permite fusionar virtualmente dos espacios reales, el living- comedor de su casa y el living-comedor de la casa que está en origen. Las personas que están presentes en ambos lugares participan de este espacio virtual sin constricción de tiempo.

\section{Comentarios finales}

Los procesos migratorios y sus implicancias sociales, culturales, económicas y políticas, han sido abordados desde diversas perspectivas. Bajo el enfoque trasnacional, se han llevado a cabo estudios sobre la cons- 
trucción de familias y maternidades trasnsfronterizas. En el caso de las familias, son diversas las investigaciones que dan cuenta de las transformaciones que ésta experimenta, abriendo un importante debate en torno a nuevas formas de organizar y construir familias más allá de los límites territoriales. Parte importante de la literatura ha buscado comprender las múltiples formas en que los miembros de las familias de inmigrantes que viven translocalmente, mantienen los vínculos afectivos y de parentesco, pese a la distancia que separa a sus miembros. El presente artículo buscó analizar la relación que existe entre estas prácticas y el lugar donde estas se realizan. De este modo se buscó comprender de manera localizada y microsocial, las dinámicas, práctica y relaciones sociales trasnacionales.

Uno de los resultados del estudio fue comprender que las mujeres suelen volver al mismo lugar para realizar las llamadas telefónicas, lo que significa que se produce una rutinización de la práctica. El ciber se transforma de este modo en el lugar desde donde se ejerce la maternidad a distancia.

Otro aspecto observado es que cuando las mujeres van a los centros de llamadas a comunicarse con sus hijos y familiares, se va produciendo un tipo particular de uso de los espacios públicos, pues ellas acceden a estos espacios de manera funcional. La calle Catedral genera en varias mujeres sospecha, no les agrada ver a las personas ahí sentadas, 'esperando que les llegue un trabajo’. Es por ello que se aproximan a este lugar en forma más utilitaria, se trata de ir a llamar y regresar, entrar y salir, no quedarse, pues hay temor a ser confundida con aquello que rechazan. A diferencia de los hombres que habitan la esquina y que la han hecho suya, la relación de algunas mujeres con este lugar es de tránsito, de pasada, tiempos cortos y breves cargados se emoción y significados, pero que no le permiten adueñarse de lo público. 


\section{Notas}

${ }^{1}$ Una versión preliminar de este artículo fue presentado en el seminario "El Impacto de la Migración en la Familia”. Universidad de los Andes, 22 agosto 2012.

${ }^{2}$ Académica Departamento Sociología, Universidad Alberto Hurtado. Este estudio se enmarca dentro del proyecto Fondecyt $N^{\circ} 1110414$. "La construcción del espacio y del lugar en contextos de migración trasnacional. Espacios públicos y espacios privados de la migración peruana en Chile”.

${ }^{3}$ Proyecto Fondecyt $N^{\circ} 1110414$. Los nombres de los entrevistados fueron modificados para mantener la confidencialidad.

${ }^{4}$ El Caracol de Catedral es una galería comercial que hasta mediados de los noventa evidenciaba un claro abandono y deterioro. Sin embargo, desde el 2000 en adelante comenzó a ser repoblada por negocios de inmigrantes y hoy día más del 90\% de los locales comerciales son administrados por extranjeros y orientados al público inmigrante.

${ }^{5}$ Los inmigrantes hombres que buscan trabajo en la construcción se concentran en una de las esquinas de la calle Catedral.

${ }^{6}$ Las formas de apropiación de la esquina de Catedral por parte de los inmigrantes en Santiago centro se presentó en el seminario: Migración latinoamericana: el papel del Estado y la sociedad en el proceso de Integración, realizado en Santiago de Chile, el 23 de Agosto de 2012. La ponencia se tituló: "La construcción del lugar y los significados del enclave” y el artículo se encuentra en preparación. 


\section{Bibliografía}

Bryceson, Deborah y Vuorela, Ulla (2002), The transnational family. New European frontiers and global network. Ediciones Berg, USA.

Glick Schiller, Nina y Çaglar, Ayse (2011), “Downscaled Cities and Migrants Pathways: Locality and Agency without and Ethnic Lens”. En: Glick Schiller, Nina y Çagalar, Ayse, Locating Migration. Rescaling Cities and Migrants. Cornell University Press, p.279. ISBN 978-0-8014-7687-7, Nueva York.

Hondagneu-Sotelo, Pierrete Doméstica (2007), Immigrant workers cleaning and caring in the shadows of affluence, University of California Press, USA.

Hondagneu-Sotelo, Pierrete, Avila, Ernestina (1997), “I'm here, but I'm there”. En: Gender and Society, Vol 11, N5. Pp.548-571. Sage Publication. http://links.jstor.org/sici?sici=0 $8912432 \% 28199710 \%$ 2911\%3А5\%3C548\%3А\%22HBITT\%3E2.0.CO\%3B2-I

Levitt, Peggy y Glick Schiller, Nina (2008), “Conceptualizing Simultaneity: A Transnational Social Field Perspective on Society”. En: Khagram, Sanjeev y Levitt, Peggy, The Transnational Studies Reader. Intersections and Innovations, Routledge, 284-294. 978-0-415-95372-6, Nueva York.

Lee, Sarah (1999), "Private users in public spaces. A study of an Internet café”. En: New Media Society, 1:331, Sage Publications.

Levitt, Peggy (1998), "Social Remittances: Migration Driven Local-Level Forms of Cultural Diffusion”. En: International Migration Review, vol 32, N4 (Winter 1998). http://www.jstor.org/stable/2547666. Acceso 05/12/2011.

Martínez, Luz María; Peñaranda, María Carmen; Vitores, Anna (2011), "Los locutorios como espacios de integración: las tecnologías de la información y la comunicación en la construcción de redes e identidades”. Revista Psicoperspectivas. Individuo y sociedad. Vol. 10 N¹, pp. 243-270.

Madianou, Mirca y Miller, Daniel (2011), “Mobile phone parenting: Reconfiguring relationships between Filipina migrant mother and their left behind children”. En: New Media Society 13:457, Published online 23 march 2011, Sage Publications.

Parrado Emilio, Flippen, Chenoa (2005), “Migration and gender among Mexican Women”. En: American Sociological Review, Vol. 70, N4, Agosto 2005, pp. 606-632. http://www.jstor.org/stable/4145379 Acceso $06 / 08 / 2008,14: 35$.

Peñaranda, María Carmen; Vitores, Anna; Martínez, Carmen (2011), “El acceso público a las tecnologías de la información t comunicación: el lugar de los locutorios en los process migratorios”. Papeles del CEIC 
N 70, marzo 2011, ISSN: 1695-6494.

Pessar, Patricia y Mahler, Sarah (2003), “Transnational Migration: Bringing Gender”. En: International Migration Review, Vol. 37, No. 3, (Fall, 2003), pp. 812-846: http://www.jstor.org/stable/30037758. Acceso: 01/12/2009.

Pries, Ludger (2005), “Configurations of geographic and societal spaces: a sociological proposal between 'methodological nationalism' and the 'spaces of flows’ ”. En: Global Networks 5, 2, 167-190. ISSN 1470-2266.

Ritzer, George (2000), The mcdonalization of society. New century Ed., USA.

Salazar Parreñas, Rhacel (2003), “The care crisis in the Philippines: Children and Transnational families in the new global economy”, en: Ehrenreich, Barbara y Russel Hochschild, Arlie (s/d), Global Women. Nannies, Maids and Sex Workers in the New Economy. Metropolitan Books, New York.

Sassen, Saskia (2001), The Global City. New York, London, Tokio. 2a Edición. Princeton University Press. Pp.398.

Schmalzbauer, Leah (2004), "Searching for wages and mothering from afar: The case of Honduran transnational families". En: Journal of Marriage and Family, Vol 66, N5. Special Issue: Intenational Perspectives on Families and Social Change. Dec 2004, pp.1317-1331.

Ídem (2008), Family divided: the class formation of Honduran transnational families. Global Networks 8,3, 329-346.

Senyurekli, Aysem; Detzner, Daniel (2008), “Intergenerational relationships in a transnational contexts: The case of Turkish families”. En: Family Relations. Interdisciplinary Journal of Applied family studies, Vol 66, $\mathrm{N}^{\circ}$ 57, pp. 457-467.

Smith, Michael Peter (2003), Transnational Urbanism. Locating globalization, 3a edición. MA Estados Unidos. Blackwell Publishing. P.221. ISBN: 0-631-18423-6.

Takanishi, Ruby (2004), “Leveling the playing field: Supporting Immigrant Children from Birth to Eight”. En: The future of Children, Vol $14 \mathrm{~N}^{\circ} 2$, Children of immigrant Families /Summer 2004) pp 60-74.

Parella, Sonia (2007), “Los vínculos afectivos y de cuidado en las familias transnacionales Migrantes ecuatorianos y peruanos en España”. En: Revista Migraciones Internacionales, Vol 4, Num 2, Jul.Dic 2007. 